\title{
Rickettsia honei sp. nov., the aetiological agent of Flinders Island spotted fever in Australia
}

\author{
John Stenos, ${ }^{1}$ Véronique Roux, ${ }^{2}$ David Walker ${ }^{1}$ and Didier Raoult ${ }^{2}$
}

\author{
Author for correspondence: David Walker. Tel: + 1409772 2856. Fax: + 14097722500. \\ e-mail: dwalker@marlin.utmb.edu
}

\begin{abstract}
1 Department of Pathology, University of Texas Medical Branch, 301 University Blvd, 1.116 Keiller Bldg, Galveston, TX 77550-0609, USA

2 Unité des Rickettsies, CNRS UPRESA 6020, Faculté de médecine, 27 Bd Jean Moulin, 13385 Marseille, France
\end{abstract}

\begin{abstract}
The name Rickettsia honei, strain RB', has been proposed for a unique spotted fever group (SFG) agent which is pathogenic for humans. This agent has previously been compared to the other SFG agents and was shown to be distinct in protein structure by SDS-PAGE and by immunoblotting. Genetic comparisons of the 165 rRNA, rompA, g/tA and the $17 \mathrm{kDa}$ antigen genes with the other SFG rickettsiae confirmed the phylogenetic distance between $R$. honei and the previously described species. Genetically, Rickettsia honei is more closely related to the Thai tick typhus (TT-118) rickettsia than to any other member of the SFG. Indeed, it is proposed that TT-118 is a strain of $R$. honei which was previously isolated in Thailand. These results elucidate the presence of a unique SFG rickettsial species in Australasia.
\end{abstract}

Keywords: Rickettsia honei sp. nov., spotted fever group

\section{INTRODUCTION}

The human disease, Queensland tick typhus (QTT), which is tick transmitted and is caused by Rickettsia australis, was believed to be limited to the Australian states of Queensland and New South Wales. However, recent reports have described spotted fever group (SFG) rickettsial infections occurring in the southeastern region of Victoria (6), Flinders Island (28) and Tasmania (5).

An SFG rickettsiosis-like ailment was identified on Flinders Island in 1991 by the local medical practitioner, Dr Robert Stewart, who suspected the clinical symptoms of the patients to be rickettsial in origin (28). Signs and symptoms of the disease included fever, headache, myalgia, slight cough and a maculopapular rash. Serological analysis, including the Weil-Felix agglutination and rickettsial-specific immunofluorescence (IF) tests, suggested that the causative agent was a member of the SFG (10).

Blood was collected from patients presenting with Flinders Island spotted fever (FISF) prior to antibiotic therapy. Concentrated buffy coat from the blood was inoculated onto Vero cell monolayers, which were subsequently centrifuged (26). Weekly cell culture

Abbreviations: FISF, Flinders Island spotted fever; IF, immunofluorescence; SFG, spotted fever group.

The GenBank accession numbers of the $R$. honei gene sequences in this study are AF018074 (g/tA), AF018075 (rompA $590 \mathrm{bp}$ fragment) and AF018076 (rompA 3176 bp fragment). scrapings of the monolayer were examined by IF, and on the fourth week a microbe of typical rickettsial morphology was observed (11).

There was no apparent cytopathic effect in the primary isolation. On subsequent passages, cytopathic effect was observed and was characterized by scattered irregularly shaped lesions with cells detaching from the monolayer (1). Electron microscopy revealed a Gramnegative bacillary bacterium measuring $0.4 \mu \mathrm{m}$ in diameter and $1.0 \mu \mathrm{m}$ in length, and having a lowdensity cytosol and encompassed by a multilayered outer membrane which was loosely adherent to the inner membrane (1).

At the genetic level, the $17 \mathrm{kDa}$ antigen and the $16 \mathrm{~S}$ rRNA genes of Rickettsia honei have been sequenced and compared with other members of the SFG. The analysis revealed a unique set of sequences for this agent, grouping it with Rickettsia rickettsii and Rickettsia slovaca (2). Subsequently, there have been several more sequence additions to the $17 \mathrm{kDa}$ antigen and 16S rRNA gene database, and hence the phylogenetic position of $R$. honei needs to be re-evaluated. We further characterize the genetic position of the FISF agent by citrate synthase $(g l t A)$ and rickettsial outer-membrane protein A $(\operatorname{romp} A)$ gene sequence analyses. These two genes have been sequenced for most of the other members of the SFG rickettsiae and were shown to be useful tools to study the phylogenetic organization of these obligately intracellular bacteria $(9,23)$. On the basis of our results together with the previous publications on the geographic isolation, 
genetic sequencing, phenotypic and protein analysis of this agent, we formally propose the name Rickettsia honei, the type strain of which is $\mathrm{RB}^{\mathrm{T}}$.

\section{METHODS}

Cell culture. $R$. hone $i$ was seeded into confluent Vero cell monolayers in RPMI 1640 medium as described previously (11). The infected cell culture was harvested, and the rickettsiae purified using renografin density-gradient centrifugation (27).

Rickettsial chromosomal DNA extraction. Purified $R$. honei were incubated with $1 \mathrm{mg}$ proteinase $\mathrm{K} \mathrm{m}^{-1}$ and $1 \%$ SDS at $56{ }^{\circ} \mathrm{C}$ for $1 \mathrm{~h}$. This digestion was followed by two phenol/ chloroform (50:50) extractions and ethanol precipitation. The resultant DNA pellet was washed with $70 \%$ ethanol, air-dried and reconstituted with nuclease-free water.

PCR amplification of the $R$. honei gitA and rompA. Oligonucleotides were designed from the sequence of the $R$. rickettsii glt $A$ gene with the exception of RpCS1258, which has been described previously (Table 1) (20). The $R$. honei glt $A$ was amplified using $2 \mu \mathrm{mol}$ primer pair CS-162-F and RpCS1258, $200 \mu \mathrm{mol}$ dNTPs and $10 \mathrm{ng} R$. honei DNA in Advantage Tth Polymerase mix (Clontech). The amplifi-

Table 1. Primer sequences of the rickettsial gltA gene

\begin{tabular}{|ll|}
\hline \multicolumn{1}{|c|}{ Primer } & \multicolumn{1}{c|}{ Primer sequence } \\
\hline CS-162-F & 5' GCA AGT ATC GGT GAG GAT GTA ATC 3' \\
CS-398-SF & 5' ATT ATG CTT GCG GCT GTC GG 3' \\
CS-764-SF & 5' ATT GCC TCA CTT TGG GGA CC 3' \\
RpCS1258 & 5'ATT GCA AAA AGT ACA GTG AAC A 3' \\
CS-731-SR & 5'AAG CAA AAG GGT TAG CTC C 3' \\
CS-411-SR & 5' AAT GCC GCA AGA GAA CCG ACA G 3' \\
\hline
\end{tabular}

Table 2. GenBank accession numbers of rickettsial sequences used in this study

\begin{tabular}{|c|c|c|c|c|c|}
\hline \multirow[t]{2}{*}{ Organism } & \multirow[t]{2}{*}{ Strain } & \multicolumn{4}{|c|}{ Accession no. for: } \\
\hline & & $\begin{array}{l}17 \mathrm{kDa} \\
\text { antigen }\end{array}$ & 16S rDNA & glt $A$ & ompA \\
\hline Rickettsia parkeri & Maculatum 20 & U17008 & L36673 & U59732 & U43802, U83449 \\
\hline Rickettsia sibirica & 246, ATCC VR-151 ${ }^{\mathrm{T}}$ & & L36218 & U59734 & U43807, U83455 \\
\hline 'Rickettsia mongolotimonae' & HA-91 & & L36219 & U59731 & U43796, U83439 \\
\hline Strain $\mathrm{S}$ & Strain $\mathrm{S}$ & & U25042 & U59735 & U43805, U83452 \\
\hline Rickettsia africae & ESF-5 & & L36098 & U59733 & U43790, U83436 \\
\hline Rickettsia honei & $\mathrm{RB}^{\mathrm{T}}$ & M99391 & U17645 & AF018074 & AF018075, AF018076 \\
\hline Thai tick typhus rickettsia & TT-118, ATCC VR-599T & AF027124 & L36220 & U59726 & U43809, U83456 \\
\hline Astrakhan fever rickettsia & A-167 & & L36100 & U59728 & U43791, U83437 \\
\hline Israeli tick typhus rickettsia & ISTT CDCl & & L36223 & U59727 & U43797, U83441 \\
\hline Rickettsia conorii & Moroccan, ATCC VR-141 & M28480 & L36105 & U59730 & U43244, U83448 \\
\hline Rickettsia rickettsii & $\mathrm{R}$ (Bitterroot), ATCC VR-89 & M16486 & L36219 & U59729 & U43804, U83451 \\
\hline 'Rickettsia slovaca' & $13-B$ & & L36224 & U59725 & U43808, U83454 \\
\hline Rickettsia japonica & YM & D16515 & L36213 & U59724 & U43795, U83442 \\
\hline Rickettsia massiliae & Mtu1 & & L36214 & U59719 & U43799, U83445 \\
\hline Rickettsia rhipicephali & $3-6-7$ & U11020 & L36216 & U59721 & U43803, U83450 \\
\hline Rickettsia aeschlimannii & MC16 & & U74757 & U59722 & U43800, U83446 \\
\hline Rickettsia montanensis & M/5-6 & U11017 & L36215 & U74756 & U43801, U83447 \\
\hline Rickettsia helvetica & С9P9 & & L36212 & U59723 & \\
\hline Rickettsia akari & MK (Kaplan), ATCC VR-148 & & L36099 & U59717 & \\
\hline Rickettsia australis & Phillips & M74042 & L36101 & U59718 & \\
\hline Rickettsia typhi & Wilmington, ATCC VR-144 & M28481 & L36221 & U59714 & \\
\hline Rickettsia prowazekii & Breinl, ATCC VR-142T & M28482 & M21789 & M17149 & \\
\hline $\mathrm{AB}$ bacterium & & U04162 & U04163 & U59712 & \\
\hline Rickettsia canadensis & 2678, ATCC VR-610 & M82879 & L36104 & U59713 & \\
\hline 'Rickettsia amblyommii' & MO 85-1084 & U11013 & & & \\
\hline 'Rickettsia felis' & & M82878 & & & \\
\hline Rickettsia bellii & $369 L 42-1$ & U11013 & L36103 & U59716 & \\
\hline Bartonella quintana & Fuller, ATCC VR-358 & M82878 & M73228 & L38987 & \\
\hline
\end{tabular}


cation was performed on a thermal cycler (Perkin Elmer Cetus) with 35 cycles of denaturation at $95^{\circ} \mathrm{C}$, annealing at $48^{\circ} \mathrm{C}$ and extension at $70^{\circ} \mathrm{C}$ for $1 \mathrm{~min}$ intervals. The resultant PCR product was purified from unincorporated primers and nucleotides using the QIAquick PCR purification kit (Qiagen). Amplification of rompA was performed as described previously (9).

DNA sequencing of PCR products. The purified glt $A$ and romp $A$ PCR products were sequenced using the ABI PRISM 377 Sequencer (Perkin Elmer) and ALF DNA sequencer (Pharmacia LKB), respectively.

DNA analysis. The gene sequences of the $16 \mathrm{~S}$ rRNA, $17 \mathrm{kDa}$, $g l t A$ and romp $A$ for the rickettsial species were extracted from GenBank (Table 2). The homologous rickettsial gene segments were aligned with those of other rickettsial sequences using the multisequence alignment program CLUSTAL. Phylogenetic relationships were inferred using the PHYLIP version 3.4 software package (J. Felsenstein, University of $\mathrm{W}$ ashington, Seattle, USA). Evolutionary distance matrices, generated by DNADIST, were determined by the methods of Kimura (16). These matrices were used to infer dendrograms using the neighbour-joining method (24). The data were also examined using the parsimony analysis (DNAPARS). The stability of predicted branching orders was assessed by generation of a consensus tree from 100 trees derived from bootstrap samples of the original alignment using SFQBOOT and CONSENSE in the same package.

\section{RESULTS AND DISCUSSION}

\section{Genetic analysis}

Phylogenetic trees derived from distance matrix analysis generated from $16 \mathrm{~S} \mathrm{rRNA}$, glt $A$, romp $A$ and $17 \mathrm{kDa}$ antigen gene sequence alignments are presented in Figs

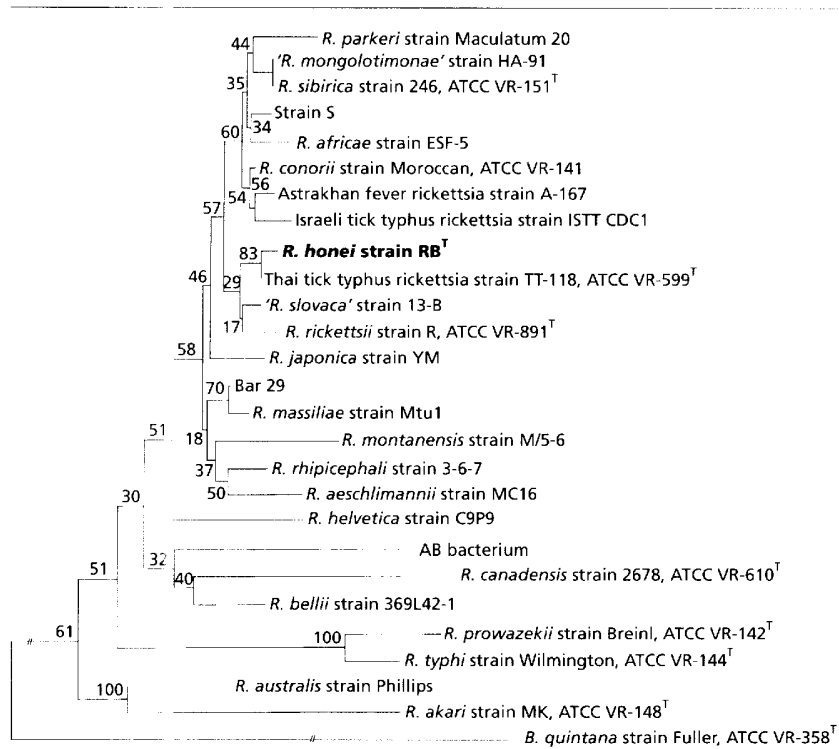

$0.5 \%$ of difference

Fig. 1. Phylogenetic tree obtained by a neighbour-joining analysis of the 16S rRNA gene sequences, showing the position of $R$. honei in the $\alpha-1$ subclass of the Proteobacteria. Bootstrap values from 100 analyses are shown at the branch points of the tree. The accession numbers of the sequences of the organisms used in the analysis are listed in Table 2.

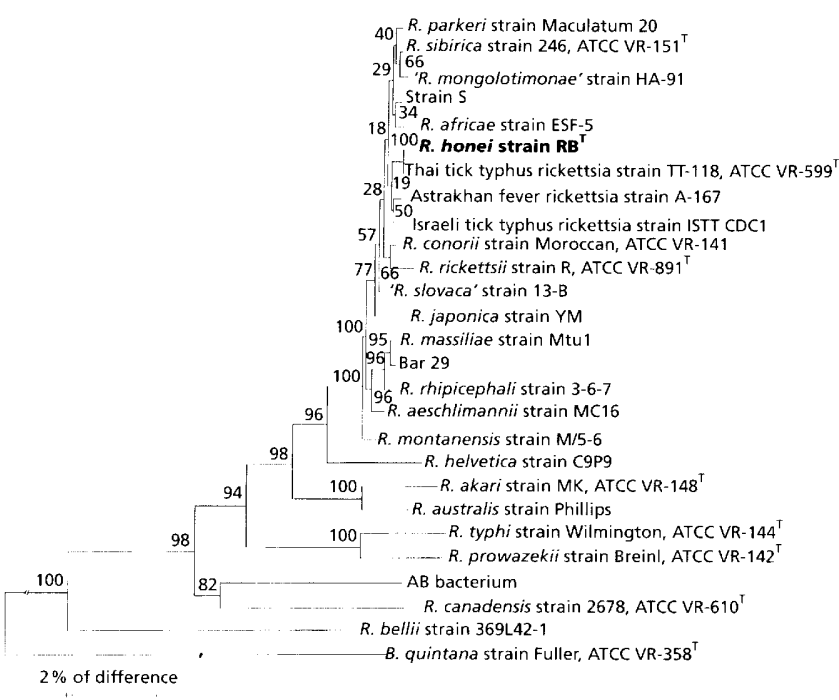

Fig. 2. Phylogenetic tree obtained by a neighbour-joining analysis of the $g / t A$ sequences.

$1,2,3$ and 4, respectively. The general organization of the four dendrograms was the same within the trees derived from parsimony analysis as that found in evolutionary distance matrix analysis. However, branching order for each gene was slightly different especially when bootstrap values were low. In all four phylodendrograms, $R$. honei and the Thai tick typhus rickettsia (TT-118) are grouped together in a unique branch which is closely associated to the core SFG branch but far from $R$. australis. In fact these two agents had identical gene sequences except for the $16 \mathrm{~S}$ rDNA gene where they differed by 1 nucleotide. Trees generated using the $17 \mathrm{kDa}$ antigen gene by parsimony and distance matrix analysis revealed low bootstrap values. This situation indicated that this particular gene is not a suitable tool for phylogenetic analysis. However, this phylogenetic tree, like the others, does display a similar relationship between $R$. honei and the other SFG members. As previously determined, romp $A$ sequence comparison is the best tool to infer phylogenetic relationships among the SFG rickettsiae which diverged late from the common ancestor of this group of bacteria (9). Our data support this opinion since the bootstrap values are the greatest for this phylogenetic tree, in most cases equalling 100 .

The plethora of rickettsial genetic information currently available in GenBank, including 16s rRNA, $17 \mathrm{kDa}$, glt $A$ and romp $A$ genes, has helped with the speciation of several human and tick rickettsial isolates including Rickettsia massiliae and Rickettsia aeschlimannii $(3,4)$.

\section{Justification of a new species}

Traditionally, microimmunofluorescence using a particular protocol for developing mouse antisera against the SFG rickettsiae has been the reference method for defining the relationships between taxa of the SFG 


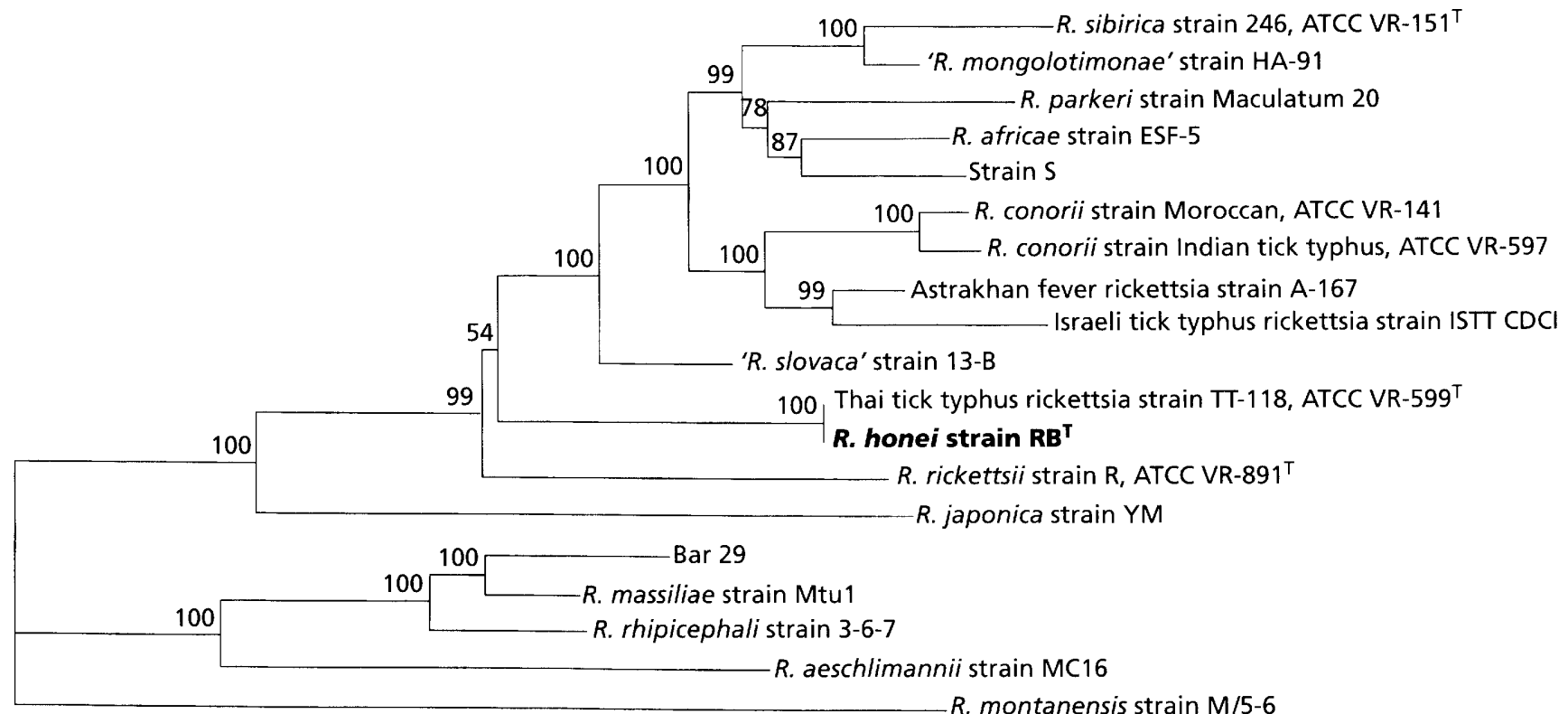

R. montanensis strain $\mathrm{M} / 5-6$

Fig. 3. Phylogenetic tree obtained by a neighbour-joining analysis of the rompA sequences.

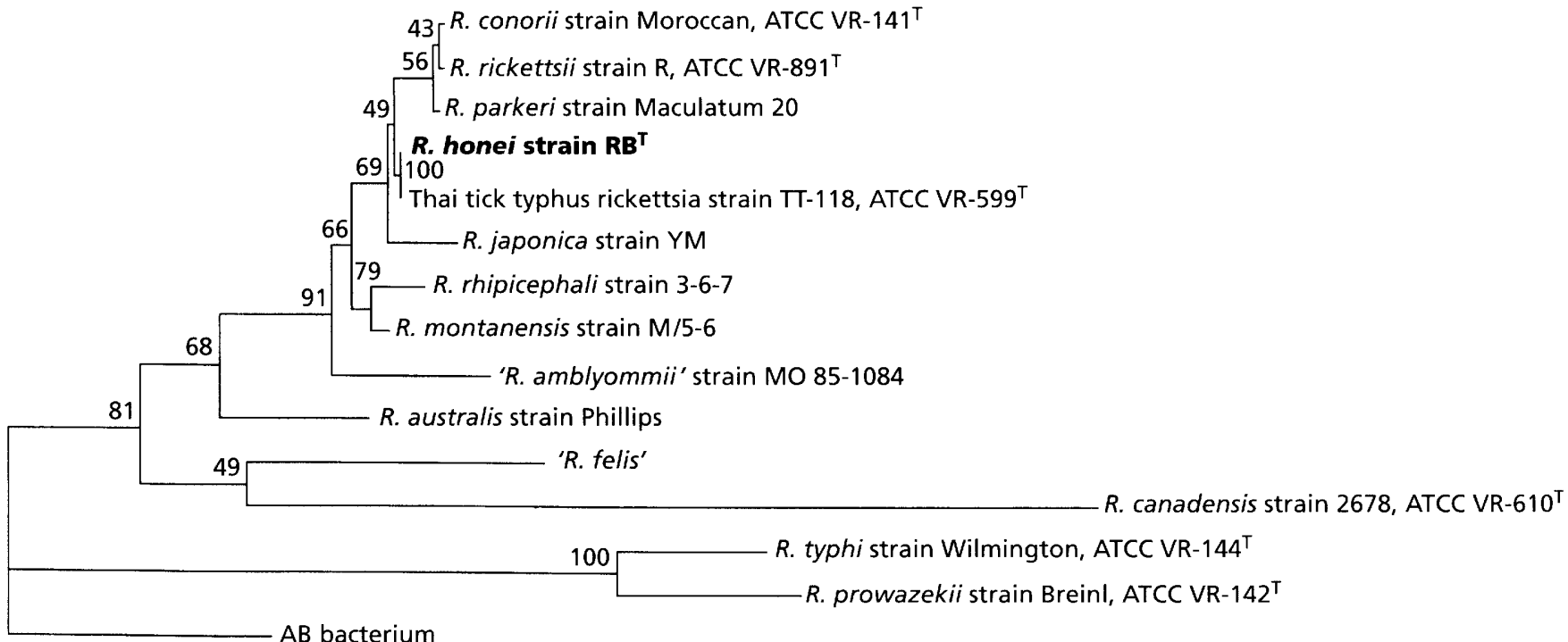

$2 \%$ of difference

Fig. 4. Phylogenetic tree obtained by a neighbour-joining analysis of the $17 \mathrm{kDa}$ antigen gene sequences.

rickettsiae $(18,29)$. However other assay systems including SDS-PAGE, immunoblotting and gene sequencing are more objective methods that can be utilized to determine the phylogenetic identity of a particular agent (19).
SDS-PAGE and immunoblot analysis of $R$. australis and $R$. honei protein lysates revealed differences in protein profiles and structure. There were distinct differences between these rickettsiae in terms of protein electrophoretic mobility and antigenicity. Further 
analysis with species-specific monoclonal antibodies derived from other members of the SFG showed no reactivity with $R$. honei (27).

Although the 16S rRNA gene has been used for the speciation of bacterial isolates, it has been proposed that sequence homologies greater than $97 \%$ should rely on DNA-DNA reassociation for taxonomic classification (25). The SFG rickettsiae share more than $97.8 \%$ homology in the $16 \mathrm{~S}$ rRNA gene, and with the exceptions of Rickettsia akari and $R$. australis DNA-DNA homology analysis groups the rest of the spotted fever group members into a single species (22, $30)$. However, it has been proposed that the evolutionary selective pressures of the rickettsiae may differ from the enterobacteria due to the differences in genome size and strict intracellular growth and thus the criteria for classification should be different (15).

Based on the calibration of rRNA gene evolution by Ochman \& Wilson (17), the divergence of $R$. honei and $R$. australis occurred 65 million years ago whereas the divergence of $R$. honei and TT-118 has occurred recently. It seems plausible that the divergence of the common ancestors of $R$. australis and $R$. honei can be explained by continental drift. The close relationship of $R$. hone $i$ and TT-118 suggests that they are strains of the same species. Although the vector ecology of $R$. honei and TT-118 is not clearly defined and both strains are found in Australasia, they may have been introduced to one site or the other by migratory birds. For example, the Curlew sandpiper (Calidris ferruginea), Sanderling (Calidris alba) and black-bellied plover (Pluvialis squatarola) migrate south from the Arctic tundras through Asia to coastal regions of Australia (7). The arthropod that harbours $R$. honei has not been identified, but a reptile-associated tick namely, Aponoma hydrosauri, has been suspected based on temporal association of bite with onset of illness in a patient (11). TT-118 was isolated from a mixed pool thought to represent larval Ixodes sp. and Rhipicephalus sp. (21). This situation would suggest that the rickettsial species may have been introduced by a rickettsemic bird or a tick carried upon a bird. We propose that $R$. honei (strain $\mathrm{RB}^{\mathrm{T}}$ ) be designated as the reference strain since it is an isolate from a patient who displayed the clinical symptoms of SFG rickettsial disease (11). The likeness of these two strains will be investigated further by microimmunofluorescence and monoclonal antibody typing.

Recent publications have shown the distinctiveness of $R$. hone $i$, both at the protein and genetic levels $(1,2$, 27 ). We have pooled the previously available genetic information together with the glt $A$ and romp $A$ sequences to show this agent's relationship to the other SFG members. Characteristically the SFG members are all genetically closely related to one another, but have been assigned species names due do their geographic isolation and diverse arthropod host life cycles. Both rickettsial glt $A$ and rompA genes have been proposed as phylogenetic tools for determining the relationships among the rickettsiae $(8,23)$. We have used these genes together with the $17 \mathrm{kDa}$ antigen and $16 \mathrm{~S}$ rRNA genes to demonstrate that $R$. honei is a unique species of the SFG rickettsiae.

\section{Description of Rickettsia honei sp. nov.}

Rickettsia honei [ho.ne.i. L. gen. n. honei of Hone, named after Frank Sandland Hone, an Australian physician who was an early pioneer in Australian rickettsiology $(8,12-14)]$.

This agent is an obligately intracellular bacterium which can be grown at $32{ }^{\circ} \mathrm{C}$ in Vero cells with RPMI 1640 supplemented with $10 \%$ foetal calf serum and $2 \mathrm{mM}$ L-glutamine (11). Its morphology is characteristically rickettsial in nature as determined by electron microscopy (1). SDS-PAGE, immunoblotting and species-specific monoclonal antibody analyses demonstrated the uniqueness of $R$. honei (27). We have further characterized this agent by sequence analysis and have shown that $R$. honei is truly a new member of the SFG. The type strain of $R$. honei is strain $\mathrm{RB}^{\mathrm{T}}$, isolated from a person with the clinical manifestations of Flinders Island spotted fever. This strain has been deposited in the American Type Culture Collection as ATCC VR-1472 .

\section{ACKNOWLEDGEMENTS}

The authors wish to thank Josie Ramirez for expert secretarial skills and Bernadette Thiers for her technical assistance. This work was supported in part by a grant from the National Institute of Allergy and Infectious Diseases (AI 21242).

\section{REFERENCES}

1. Baird, R. W., Lloyd, M., Stenos, J., Ross, B. C., Stewart, R. S. \& Dwyer, B. (1992). Characterization and comparison of Australian human spotted fever group rickettsiae. $J$ Clin Microbiol 30, 2896-2902.

2. Baird, R. W., Stenos, J., Stewart, R., Hudson, B., Lloyd, M., Aiuto, S. \& Dwyer, B. (1996). Genetic variation in Australian spotted fever group rickettsiae. $J$ Clin Microbiol 34, 1526-1530.

3. Beati, L., Meskini, M., Thiers, B. \& Raoult, D. (1997). Rickettsia aeschlimannii sp. nov., a new spotted fever group rickettsia associated with Hyalomma marginatum ticks. Int $J$ Syst Bacteriol 47, 548-554.

4. Beati, L. \& Raoult, D. (1993). Rickettsia massiliae sp. nov., a new spotted fever group rickettsia. Int $J$ Syst Bacteriol 43, 839-840.

5. Chin, R. H. \& Jennens, I. D. (1995). Rickettsial spotted fever in Tasmania [letter]. Med $J$ Aust 162, 669.

6. Dwyer, B. W., Graves, S. R., McDonald, M. I., Yung, A.P., Doherty, R. R. \& McDonald, J. K. (1991). Spotted fever in East Gippsland, Victoria: a previously unrecognised focus of rickettsial infection. Med $J$ Aust 154, 121-125.

7. Elphick, J. (1995). The atlas of bird migration. Tracing the great journeys of the world's birds, pp. 128-129. New York: Random House.

8. Fenner, F. (1990). History of microbiology in Australia. In Rickettsioses, pp. 265-266. Maryborough, Victoria: Australian Print Group. 
9. Fournier, P. E., Roux, V. \& Raoult, D. (1998). Phylogenetic analysis of spotted fever group rickettsiae by study of the outer surface protein rOmpA. Int $J$ Syst Bacteriol 48, 839-849.

10. Graves, S. R., Dwyer, B. W., McColl, D. \& McDade, J. E. (1991). Flinders Island spotted fever: a newly recognised endemic focus of tick typhus in Bass Strait. 2. Serological investigations. Med J Aust 154, 99-104.

11. Graves, S. R., Stewart, L., Stenos, J., Stewart, R. S., Schmidt, E., Hudson, S., Banks, J., Huang, Z. \& Dwyer, B. (1993). Spotted fever group rickettsial infection in south-eastern Australia: isolation of rickettsiae. Comp Immunol Microbiol Infect Dis 16, 223-233.

12. Hone, F. S. (1922). A series of cases closely resembling typhus fever. Med $J$ Aust 1, 1-13.

13. Hone, F. S. (1923). A further series of cases closely resembling typhus fever. Med J Aust 1, 435-443.

14. Hone, F. S. (1927). Endemic typhus fever in Australia. Med J Aust 2, 213-226.

15. Kelly, P. J., Beati, L., Mason, P. R., Matthewman, L. A., Roux, V. \& Raoult, D. (1996). Rickettsia africae sp. nov., the etiological agent of African tick bite fever. Int $J$ Syst Bacteriol 46, 611-614.

16. Kimura, M. (1980). A simple method for estimating evolutionary rate of base substitutions through comparative studies of nucleotide sequences. $J$ Mol Evol 16, 111-120.

17. Ochman, H. \& Wilson, A. C. (1987). Evolution in bacteria: evidence for a universal substitution rate in cellular genomes. J Mol Evol 26, 74-86.

18. Phillip, R. N., Casper, E. A., Burgdorfer, W., Gerloff, R. K., Hughes, L. E. \& Bell, E. J. (1978). Serologic typing of rickettsiae of the spotted fever group by indirect immunofluorescence. I Immunol 121, 1961-1968.

19. Raoult, D. \& Roux, V. (1997). Rickettsioses as paradigms of new or emerging infectious diseases. Clin Microbiol Rev 10, $694-719$.
20. Regnery, R. L., Spruill, C. L. \& Plikaytis, B. D. (1991). Genotypic identification of rickettsiae and estimation of intraspecies sequence divergence for portions of two rickettsial genes. $J$ Bacteriol 173, 1576-1589.

21. Robertson, R. G. \& Wisseman, C. L., Jr (1973). Tick-borne rickettsiae of the spotted fever group in west Pakistan. $\mathrm{Am}$ $J$ Epidemiol 97, 55-64.

22. Roux, V. \& Raoult, D. (1995). Phylogenetic analysis of the genus Rickettsia by $16 \mathrm{~S}$ rDNA sequencing. Res Microbiol 146, 385-396.

23. Roux, V., Rydkina, E., Eremeeva, M. \& Raoult, D. (1997). Citrate synthase gene comparison, a new tool for phylogenetic analysis, and its application for the rickettsiae. Int $J$ Syst Bacteriol 47, 252-261.

24. Saitou, N. \& Nei, M. (1997). The neighbor-joining method: a new method for reconstructing phylogenic trees. Mol Biol Evol 4, 406-425.

25. Stackebrandt, E. \& Goebel, B. M. (1994). Taxonomic note: a place for DNA-DNA reassociation and 16S rRNA sequence analysis in the present species definition in bacteriology. Int J Syst Bacteriol 44, 846-849.

26. Stenos, J., Graves, S. \& Dwyer, B. (1992). Quantification of Rickettsia australis. Am J Trop Med Hyg 47, 141-146.

27. Stenos, J., Ross, B., Feng, H. M., Crocquet-Valdes, P. \& Walker, D. (1997). Protein characterization of Australian spotted fever group rickettsiae and monoclonal typing of Rickettsia honei. J Clin Microbiol 35, 261-263.

28. Stewart, R. S. (1991). Flinders Island spotted fever: a newly recognised endemic focus of tick typhus in Bass Strait. 1. Clinical and epidemiological features. Med $J$ Aust 154, 94-99.

29. Uchida, T., Yu, X. J., Uchiyama, T. \& Walker, D. H. (1989). Identification of a unique spotted fever group rickettsia from humans in Japan. J Infect Dis 159, 1122-1126.

30. Walker, D. H. (1989). Rocky Mountain spotted fever: a disease in need of microbiological concern. Clin Microbiol Rev 2, 227-240. 